\title{
Alternative Perspektiven für den Marktzugang und die Landwirtschaftlichen Exporte aus den Ländern des Südens
}

Anna Crole-Rees

\section{CpenEdition}

\section{Journals}

Electronic version

URL: http://journals.openedition.org/sjep/875

DOI: $10.4000 /$ sjep.875

ISSN: 1663-9677

Publisher

Institut de hautes études internationales et du développement

\section{Printed version}

Date of publication: 1 mai 2002

Number of pages: $39-48$

ISSN: $1660-5926$

\section{Electronic reference}

Anna Crole-Rees, «Alternative Perspektiven für den Marktzugang und die Landwirtschaftlichen Exporte aus den Ländern des Südens », Schweizerisches Jahrbuch für Entwicklungspolitik [Online], 21 | 2002, Online erschienen am: 10 September 2012, abgerufen am 08 September 2020. URL : http:// journals.openedition.org/sjep/875; DOI : https://doi.org/10.4000/sjep.875 


\title{
ALTERNATIVE PERSPEKTIVEN FÜR DEN MARKTZUGANG UND DIE LANDWIRTSCHAFTLICHEN EXPORTE AUS DEN LÄNDERN DES SÜDENS
}

\author{
Anna Crole-ReEs*
}

\section{EINLEITUNG}

Der jüngste Bericht der Weltbank über die weltweite Entwicklung (Weltbank, 2001) befasst sich mit dem Kampf gegen die Armut und dem Abbau der Ungleichheiten. Er empfiehlt einen besseren Zugang zu Arbeit und Einkommen, insbesondere für die Ärmsten. Die internationale Gemeinschaft möchte dazu insbesondere den Zugang zu grösseren und reicheren Märkten fördern, das heisst, zu den Märkten der Länder mit hohem Einkommen (Weltbank, 2001).

Produktion und Handel mit Gütern und Dienstleistungen haben sich in den letzten Jahrzehnten dank den Fortschritten beim Transport, der Erhöhung der Produktivität, der veränderten Vorlieben und Anliegen der Konsumentinnen und Konsumenten in den Ländern des Nordens sowie dank der Rahmenbedingungen in den verschiedenen Ländern im Norden wie im Süden tief greifend verändert.

Dieser Beitrag will einige Aspekte der Förderung landwirtschaftlicher Exporte aus den Ländern des Südens in die Schweiz aufzeigen, besonders hinsichtlich der Möglichkeiten wie auch der Schwierigkeiten der so genannten «nachhaltigen» Labels. Der erste Teil befasst sich mit den wichtigsten aktuellen Herausforderungen im Zusammenhang mit der Erhöhung der Exporte von Produkten aus dem Süden. Der zweite Teil beschreibt die biologischen und ökologischen Labels sowie jene aus den Ländern des Südens. Und der letzte Teil dieses Beitrags schlägt einige Massnahmen zur Erhöhung der Exporte von Produkten aus dem Süden vor.

\section{DIE AKTUELLEN HERAUSFORDERUNGEN DER LÄNDER DES SÜDENS FÜR EINEN BESSEREN ZUGANG ZUR VERMARKTUNG}

«Die Schweizer sind anspruchsvolle und verwöhnte Konsumenten ${ }^{1}$ » (CBI und SIPPO, 1999): so werden die Konsumentinnen und Konsumenten in der Schweiz (und den Industriestaaten allgemein) in einer Broschüre beschrieben, welche sich an Produzenten richtet, die in die Schweiz exportieren. In den vergangenen Jahrzehnten haben sich die Konsumgewohnheiten stark verändert. Heute geben die Leute weniger als $15 \%$ von ihrem Lohn für Nahrungsmittel aus. Die Vorliebe für Exotisches hat stark zugenommen, unter anderem dank

* Konsulentin, Wirtschaft/Entwicklung, av. d'Echallens 13, 1004 Lausanne. Telefon: 021.625.64.64, EMail :<Anna_CroleRees@compuserve.com>. Mein Dank geht an Olivier Matringe für sein kritisches Lektorat und seine zahlreichen und interessanten Kommentare.

1. Aus dem Englischen: «Swiss people are spoiled and demanding consumers.» 
vermehrten Reisen und besseren Transportmitteln für die Produkte. Angesichts einer viel grösseren Zahl von Gütern aus verschiedenen Ländern, die unterschiedlich produziert und verarbeitet werden, und angesichts vieler Labels und Marken wird es schwieriger, die Kundschaft anzubinden, da sie eine so grosse Auswahl hat. Sie liebt das Neue, kann aber auch an ihren bevorzugten Produkten hängen. Sie ist sehr anspruchsvoll punkto Qualität und Frische der Produkte, aber diese müssen auch praktisch sein, damit die Vorbereitung der Mahlzeit und der Einkauf immer weniger Zeit in Anspruch nehmen. Die lückenlose Versorgung, das heisst, die gleiche Auswahl an Früchten und Gemüse zu jeder Jahreszeit, sowie die Möglichkeit, die unterschiedlichsten Mahlzeiten zubereiten zu können, sind wichtige Kriterien für die Konsumierenden. Und dank der verbesserten Kommunikationsmittel interessieren sie sich auch immer stärker für soziale und Umweltfragen.

Auch die Verteilkanäle und die Nachfrage der Importeure haben sich stark gewandelt, um mit den veränderten Konsumgewohnheiten Schritt zu halten und einer quantitativ etwas stagnierenden Nachfrage überall im Norden entgegenzutreten. Bei der Verteilung geht es heute stark in Richtung Konzentration und Konsolidierung. Zur Zeit werden in Europa $50 \%$ bis $75 \%$ aller Früchte in den Supermärkten verkauft (Rabobank, 2001). In den Auslagen finden sich immer unterschiedlichere Produkte. Bei jeder Art kann der Konsument auswählen, ob er sie verpackt oder offen kaufen will, er kann sie in unterschiedlichen Verarbeitungsstadien kaufen, und er kann unterschiedliche Marken, Produkte mit oder ohne Zusätze wie Konservierungsmittel oder Nährelemente erstehen. Die Importeure ihrerseits weiten das Angebot aus und organisieren sich neu. Aus Kostengründen werden die Importwege verkürzt. Neue Aktivitäten wie die Verpackung werden integriert. Es müssen auch grössere Mengen verarbeitet werden, um immer grössere Verteiler zu versorgen. Deshalb suchen sie eine optimale Zahl von Lieferanten, damit sie die Risiken verteilen, das ganze Jahr qualitativ gute Produkte anbieten und die Transaktionskosten verringern können. Eine weitere wichtige Änderung betrifft die Rolle der Normen und Labels. Sie haben sich von Harmonisierungs- und Standardisierungsfaktoren für die Produkte zu Marketinginstrumenten gewandelt (Reardon et al., 2001). Eine Folge davon ist die konstante Anpassung der Anforderungen an verschiedene Labeltypen und -normen, damit der Marktanteil gehalten werden kann.

Die Faktoren Wertschöpfung und Vielfalt, Präsentations- und Kaufkomfort, Exklusivität und Qualität sind im Wesentlichen im Detailhandel angesiedelt, das heisst in den Auslagen der Länder des Nordens. Zwischen dem Süden und dem Norden herrscht ein starker Wettbewerb um die Wertschöpfung. Zur Zeit werden die letzten Phasen der Präsentation meist am Ort des Konsums abgewickelt. Verpackt wird wegen der Transport- und Markenkosten in den Industriestaaten, wodurch die Produzenten gezwungen sind, ihre Ware offen zu exportieren, also mit tieferer Wertschöpfung. Im Sektor Landwirtschaft gibt es zahlreiche Bemühungen zur Halbindustrialisierung oder gar Industrialisierung, sie stossen aber auf viele Hindernisse. Die lokale Nachfrage für neue und/oder stark wertvermehrte Produkte ist im Allgemeinen wegen der mittleren oder sehr tiefen Einkommen sehr beschränkt, obwohl sich dank dem Tourismus und den Veränderungen der Ernährungsgewohnheiten in den Städten die Chancen mehren.

Die Inkohärenz der Politiken zwischen den internationalen und lokalen Märkten kann die Möglichkeiten zur Vermarktung und Verarbeitung stark behindern. Die 
lokale Verarbeitung kann durch den Import von billigeren Produkten konkurrenziert werden. Ein Beispiel ist der Import von Altkleidern aus Europa nach Westafrika, der den Ausbau der lokalen Textilindustrie stark behindert. Ein weiteres Beispiel ist der Preis des aus Europa importierten Getreides, der tiefer ist als jener der lokalen Getreidearten wie Hirse und Sorghum. Dies hemmt die Anstrengungen zur lokalen Industrialisierung und entmutigt die Suche nach neuen Produkten aus diesen lokalen Getreidearten sowie die Ausarbeitung von geeigneten Ausrüstungen (Diopp, 1999). Progressive Zolltarife bleiben ein grosses Hindernis für die Anstrengungen zur Verarbeitung vor Ort. Die Weltbank (2001) erinnert daran, dass die Importzölle einiger Industriestaaten für verarbeitete Produkte viel höher sind als für Rohprodukte. Die Europäische Union hat jetzt allerdings ihren Markt für die weniger entwickelten Länder geöffnet und alle Zollhemmnisse und Quoten aufgehoben, ausser seit Februar 2001 für Waffen. Und für einige Produkte (Reis, Bananen, Zucker) gilt eine Übergangszeit. Es ist zu hoffen, dass die anderen grossen Industriestaaten ebenfalls solche Massnahmen ergreifen, welche eine gewisse Handelsneutralität herstellen. Implizit wird damit die Ungleichheit der Ressourcen zur direkten oder indirekten Unterstützung der Produktion und Verarbeitung zwischen dem Norden und dem Süden anerkannt. Die Bauern der Europäischen Union, Japans und der USA wurden 1998 mit durchschnittlich 20'000 US-\$ unterstützt, 1999 machten die Subventionen $40 \%$ ihres Bruttoeinkommens aus (UNCTAD, 2001), während in den meisten afrikanischen Ländern nach der Liberalisierung und Privatisierung der verschiedenen Dienstleistungen vor und nach der Produktion die Unterstützung des Landwirtschaftssektors zurückging. Ferner erhielt die lokale Produktion in den Ländern des Südens wegen der Reduktion oder gar Abschaffung von Importhindernissen für Landwirtschaftsprodukte Konkurrenz durch importierte Waren.

Die Produktions- und Transaktionskosten können die Bemühungen zur Vermarktung zunichte machen, weil das Produkt auf dem Weltmarkt nicht mehr konkurrenzfähig ist. Die Transaktionskosten sind im Süden oft höher als im Norden. Man schätzt sie bei Früchten und Gemüse auf $60 \%$ des Endwertes (Rabobank, 2001). Besonders hoch sind zum Beispiel die Transportkosten innerhalb Afrikas und nach Europa. So liegt die Frachtrate von Kenia nach Europa für einen Container von 40 Fuss bei 6300 US-\$, während sie von Argentinien aus bei nur 4'000 US-\$ liegt (Lhéraut, 2001). Die lokale Transportpolitik und die Faktoren Angebot und Nachfrage beeinflussen die Kosten. Die aus Westafrika per Luftfracht exportierbaren Tonnagen sind unter ein Dutzend Hauptländer aufgeteilt, gesamthaft machen sie schätzungsweise 22'000 Tonnen pro Jahr aus, also ein Drittel dessen, was Kenia von einem einzigen Flughafen aus exportieren kann (Lhéraut, 2001).

Eine höhere Produktion kann neue Märkte erschliessen, und die Erfahrung zeigt, dass zwar eine Zunahme der Produktionsmenge möglich ist. Es gibt aber einige Hindernisse, da die Organisations- und Marktanforderungen in der Folge wachsen und zu Engpässen führen können. Die oft genannten Einschränkungen betreffen die Mobilisierung von Finanzen, fehlende Kredite, Logistik, ungenügende Verwaltung und Lieferfristprobleme. Eine Schwierigkeit, die bei einigen Exporteuren als unüberwindbar gilt, ist die Respektierung der Nahrungsmittelnormen, vor allem für Kleinproduzenten (FIDA, 2001). 


\section{Das Biolabel}

Biolabels gibt es seit den frühen 50er Jahren (Damary, 2001). Ihr Ziel war die Förderung einer umweltfreundlichen Produktion und einer Vermarktung von gesunden Produkten ohne chemische Rückstände (Damary, 2001). Zur Zeit können sich die Schweizer Konsumentinnen und Konsumenten vollständig biologisch ernähren, wenn sie bereit sind, den höheren Preis für diese Produkte zu bezahlen. Rund 2\% der in der Schweiz gekauften Nahrungsmittel und Getränke werden biologisch erzeugt, und von diesen werden fast $60 \%$ in Supermärkten verkauft (ITC, 1999. Zitiert von FiBL und Sippo, 2001). Die kurz- und mittelfristigen Aussichten für biologische Nahrungsmittel werden je nach Produkt als durchschnittlich bis gut eingeschätzt, man geht von einer Wachstumsrate von rund $10 \%$ beziehungsweise $30 \%$ aus (FiBL und Sippo, 2001).

Einige Verteiler identifizieren sich zwar mit den Prinzipien der biologischen Landwirtschaft, für die meisten aber sind sie wegen der damit verbundenen Faktoren der Wertschöpfung wirtschaftlich interessant: Ausweitung des Angebots, Image bei den Konsumierenden, Auszeichnung usw. So genannte Bioprodukte dürfen in der Schweiz und in Europa nur verkauft werden, wenn sie einige Grundkriterien erfüllen, welche durch staatliche Normen festgelegt sind. Laut Damary (2001) ist es aber besser, ein Produkt auf den Markt zu bringen, das durch ein Privatlabel zertifiziert ist. Der Schweizer Produzent kann zwischen verschiedenen Labels wählen, für den Produzenten im Südens ist es aber schwierig, eine Zertifizierung zu erhalten. Die erste Einschränkung ist die ungleiche Information. Viele Produzenten wissen nichts von den Pflichten und Möglichkeiten, obwohl einige ihrer Produkte de facto auf biologische Weise angebaut werden. Da in den meisten Ländern des Südens ein lokales Zertifizierungsorgan fehlt, das von den Importländern anerkannt ist, und da sie sich deshalb an Organisationen im Ausland, im Allgemeinen im Norden wenden müssen, steigen die Kosten beträchtlich. Und schliesslich sind auch die Kosten der Zertifizierung selber hoch (Besuche, Ausbildung für die Umstellung, Kosten für Verwaltung und Kontrolle). Bei der Produktion im Süden kommt das europäische Label ECOCERT am häufigsten vor. Es hat gegenüber dem in der Schweiz bekanntesten Label Bourgeon einige komparative Vorteile. Es fordert nur eine teilweise Umstellung des Anbaus, erlaubt den Transport per Flugzeug und verfügt über verschiedene Aussenstellen in Afrika, was Zertifizierung und Überwachung verbilligt.

$\mathrm{Zu}$ den Anforderungen für die biologische Zertifizierung kommen jene des Marktes. Erstens haben nicht alle Produkte die gleichen Aussichten auf dem Markt. Laut den Verteilern haben exotische Produkte, welche noch nicht das Niveau des Massenkonsums erreicht haben, wenig Chancen, kurzfristig in den biologischen Bereich vorzustossen, so zum Beispiel gewisse Früchte. Der Einführung des biologischen Anbaus in den Ländern des Südens muss also eine detaillierte Marktstudie vorangehen, umso mehr, als die Auszeichnung «bio» lokal selten rentabel ist, da die nationale Nachfrage nach diesen teureren Produkten sehr tief ist. Ausserdem kann die Auszeichnung manchmal knapp die tiefere Rendite und die zusätzliche Arbeit kompensieren, was zum Beispiel bei der Biobaumwolle in Westafrika der Fall ist. Zweitens sind die Anforderungen der erforderlichen Mengen und der Handelspraktiken gleich wie jene des «her- 
kömmlichen» Marktes. Und drittens bevorzugt der Konsument biologischer Nahrungsmittel im Allgemeinen ohnehin das lokale Angebot (UNCTAD, 2001).

\section{$\square$ Die «fairen» Labels}

Das Label des fairen Handels beruht nicht auf der Art der Produktion, sondern auf der Qualität der Beziehungen zwischen Produzenten und Käufern sowie auf der Art der Verwaltung der Produktionskooperative. Die Organisationen für fairen Handel verpflichten sich, den Produkten des Südens direkten Zugang zu den Märkten des Nordens zu gewähren, womit sie versuchen, die Transaktionskosten so tief wie möglich zu halten und einen fairen Preis zu erreichen. Dieser Preis muss die Grundbedürfnisse der Produzenten und die Produktionskosten decken und eine Marge für Investitionen ermöglichen. Weiter verpflichten sich die Organisationen gegenüber den Produzenten zu einer Vorauszahlung und garantieren langfristige Beziehungen und Verträge (EFTA, 1998). Die Produzenten ihrerseits verpflichten sich, sich in demokratisch verwalteten Verbänden oder Kooperativen zusammenzuschliessen und die Einkommen zu verteilen.

Anders als bei den Biolabels ist der faire Handel in der Schweiz nicht gesetzlich geregelt. Deshalb bieten mehrere Organisationen Produkte aus «fairem Handel » an, die manchmal ganz unterschiedliche Anforderungen, Kontrollen und Unterstützungsarten für die Produzenten aufweisen. Seit 1999 haben sich die meisten Akteure des fairen Handels in ein «Schweizerisches Forum für den fairen Handel» zusammengeschlossen, um die Kriterien zu harmonisieren. Es können zwei Vorgehensweisen unterschieden werden. Die erste geht von lokalen Rahmenbedingungen aus und versucht, die bestehenden Ressourcen zu verwerten. Dieses Vorgehen, bei dem die Weltläden in der Schweiz Pioniere sind, ermöglichte die Einführung von Produkten aus dem fairen Handel und von neuen exotischen Waren in der Schweiz. Bei der zweiten Vorgehensweise baut man auf der Nachfrage des Marktes auf und sucht Produzenten als Partner. Ein Beispiel dafür ist Max Havelaar. Das Vorgehen der Organisationen für fairen Handel bestimmt implizit die Kriterien, welche die Produzenten zu erfüllen haben, und die Art der Partnerschaft mit den Produzenten des Südens. Schümperli Younossian (2001) zeigt auf, dass Claro SA, eine Importzentrale für Produkte aus fairem Handel, welche die Weltläden beliefert, sich an Produzenten im Süden mit unterschiedlichen Erfahrungen und Ressourcen wendet. Die Betreuung der Kleinproduzenten, welche diesen hilft, die nötigen Kompetenzen für den Export zu erwerben, ist ein wichtiger Aspekt für Claro SA (Schümperli Younossian, 2001). Max Havelaar wendet sich eher an Produzenten, welche bereits Erfahrung im Export haben und fähig sind, regelmässig grosse Mengen in guter Qualität zu liefern. Wenn ein Produzentenverband diese anspruchsvollen Kriterien nicht erfüllen kann, ermutigt ihn die Stiftung Max Havelaar, die Hilfe einer Entwicklungsorganisation anzunehmen, und stellt gegebenenfalls den Kontakt her. Der schwierige Zugang zur Zertifizierung ist auch ein Problem für grosse Produzenten des herkömmlichen Sektors. Chiquita-Bananen zum Beispiel haben vermutlich keine grossen Chancen, die Zertifizierung Max Havelaar zu erhalten, obwohl sich Chiquita im Rahmen des Projekts «Better Banana Project» mit der NGO Rainforest Alliance zusammentat (Gassmann, 2001), um ein neues umweltfreundlicheres und sozial verantwortliches Label einzuführen.

Diese unterschiedlichen Anforderungen an die Produktion sind zum grossen Teil mit den Kommerzialisierungswegen der Produkte erklärbar. Wer Produkte in 
Weltläden kauft, entscheidet sich durch den Einkauf in diesen Läden explizit, den fairen Handel zu unterstützen. Die Stärke von Max Havelaar ist, dass es ihm gelungen ist, seine zertifizierten Produkte in den traditionellen Supermärkten zu verkaufen, wodurch der Zugang dieser Produkte leichter ist und grosse Absatzmengen möglich werden. Ausserdem ist der Mehrpreis für den Konsum nicht sehr hoch: beim Orangensaft zum Beispiel 10 Rappen pro Liter. Implizit sind Qualität und Quantität jenen der im Supermarkt verkauften herkömmlichen Produkten ebenbürtig. Deshalb sind die Güter, welche das Label Max Havelaar erhalten können, von Kooperativen erzeugte Produkte des Massenkonsums. Die Verteiler sehen, dass die Aufnahme von Gütern aus dem fairen Handel in ihr Angebot Vorteile bringt: Diversifizierung des Angebots, soziales Image, Qualitätsprodukte, wachsende Nachfrage usw. Ihr Vertrieb bringt aber für den Importeur/Verteiler zusätzliche Kosten und Risiken mit sich: Das Risiko der schwankenden Weltmarktpreise gegenüber einem festen Abnahmepreis, Lizenzkosten, manchmal tiefere Mengen als im herkömmlichen Sektor. Zur Zeit machen die Marktanteile von Max Havelaar in der Schweiz für Orangensaft 3,5\%, für Kaffee 5\% und für Bananen $15 \%$ aus, und der Umsatz lag 1999 in der Schweiz bei 62 Millionen Franken (Max Havelaar, 1999). Auf europäischer Ebene wird die gesamte Wachstumsrate des fairen Handels auf 10 bis $25 \%$ geschätzt, vor allem dank der Einführung neuer Produkte auf neuen Märkten (Ronchi, 2000). Zurzeit werden diese Produkte selten in den Ländern des Südens verarbeitet. Über $60 \%$ der 2500 Produkte, welche Fair-Trade-Labels tragen, sind Rohprodukte wie Kaffee, Tee, Honig, Kakao, Zucker und Bananen (Ronchi, 2000).

\section{Die Labels «des Südens»}

Es muss festgehalten werden, dass die meisten «nachhaltigen » Labels im Norden konzipiert wurden, und dass nur wenige Länder im Süden über öffentliche und/oder im Rahmen der Labelgesetzgebung geregelte Zertifizierungsstellen verfügen. Es gibt aber immer mehr Initiativen für eine Aufwertung lokaler Produkte.

Benin hat ein Qualitätslabel für seine Baumwollfasern geschaffen, das auf dem Weltmarkt für Fasern weit gehend anerkannt ist. Es basiert auf den eigentlichen Qualitäten der Faser wie Länge, Farbe, Unvollkommenheiten usw., integriert die Verfolgung des Wegs bis ins Ursprungsdorf und ermöglicht die Zahlung einer Prämie (oder einer Rückvergütung) in Bezug auf einen Referenzpreis, den Weltpreis $^{2}$, an die Besitzer, das heisst die Verarbeiter der Fasern. Da auch die Produktionsbedingungen einen Einfluss auf die Rahmenbedingungen haben, unterscheidet sich der dem Produzenten bezahlte Preis gemäss der Qualität der Baumwollkapseln. Aufgrund des aktuellen Preisfestsetzungssystems gibt es keine direkte Verbindung zwischen der beim Export bezahlten Qualitätsprämie und dem Preis, der dem Produzenten bezahlt wird. Ein weiteres Beispiel ist das SQMM (Safe Quality Mango Management), das die Vereinigung SAMGA (South African Mango Growers' Association) schuf, der praktisch alle Mangoproduzenten Südafrikas angehören. Dieses Label soll vor allem den Zugang von Früchten zum internationalen Markt erleichtern, der sich nach der Aufhebung des Embargos geöffnet hat (Finnemore, persönliche Mitteilung). Es umfasst auch das von der Europäischen Union geforderte Konzept HACCP (Hazard Analysis Critical Control Points) und basiert auf der integrierten Produktion. Nur Produ-

2. Index A von Liverpool. 
zenten, die akkreditierte Mitglieder sind, dürfen das Logo benutzen, ein Kleber, der auf jeder Palette mit Früchten anzubringen ist (SAMGA, 1999). Ein drittes Beispiel ist das Label Homegrown, das von einem Privatunternehmen für Gartenbauprodukte in Kenia entwickelt wurde. Es basiert ebenfalls auf der integrierten Produktion und umfasst auch soziale Aspekte (Evans, 1999). Die Stärke dieses Unternehmens liegt darin, dass es dank seines Labels in Europa «aufsteigen» konnte. Es liefert vorverpacktes Gemüse direkt an Supermärkte. 90\% seiner Produktion wird auf seinem eigenen Gebiet angebaut. Der Rest kommt von Kleinbauern, welche als Zulieferer dienen. Diese Beispiele sind ziemlich repräsentativ, weil sie auf lokale Initiativen zurückgehen, bei denen es im Wesentlichen um Exporte von traditionellen Produkten wie Kakao, Tee, Baumwolle und seit kurzem Gemüse geht. Hauptgrund dafür sind die Zertifizierungskosten und die Einführung von Zertifizierungsstellen.

\section{$\square$ Massnahmen zur Steigerung der Exporte aus dem Süden}

In den meisten Ländern in Afrika südlich der Sahara liegt das Bruttosozialprodukt pro Kopf unter 700 US-\$, also rund 55-mal unter jenem der Schweiz (Weltbank, 2001). Dank den Möglichkeiten, das Einkommen über eine Diversifizierung und eine bessere Vermarktung auf den lokalen und internationalen Märkten zu erhöhen, können Ungleichheiten abgebaut werden. Es gibt mehrere Wege, für die Produzenten im Süden den Marktzugang zu verbessern und die Möglichkeiten für die Vermarktung oder gar den Export zu nutzen.

Kohärente lokale wie internationale Politiken sind unumgänglich für einen besseren Zugang der Produkte aus dem Süden zu den regionalen und internationalen Märkten und für ein besseres Gleichgewicht der Produkteverwertung zwischen Nord und Süd. Es sind mehrere Massnahmen nötig. Die erste ist die Harmonisierung der Politiken zur Unterstützung von Produktion und Vermarktung, sowohl regional für den Süd-Süd-Handel wie international. Das gilt auch für die Vorschriften der nationalen Reglementierungsbehörden. Die Berechnung der Produktionskosten muss überdacht und zwischen Nord und Süd harmonisiert werden. Es gibt zahlreiche nicht monetäre Kosten wie die Zeit für Ausbildung und/oder Beratung, die Bezahlung der Arbeitskräfte in Naturalien (zum Beispiel Mahlzeiten), die einen grossen Teil der Gesamtkosten ausmachen können (CroleReese und Soulé, 2001). Sie müssen in die Kosten einbezogen werden, um mehr Gerechtigkeit zwischen Nord und Süd zu erreichen. Das gilt auch für die sozialen und Umweltaspekte, im Süden wie im Norden.

Die Vorlieben und Anliegen der Konsumentinnen und Konsumenten unterscheiden sich unter anderem nach Region, Periode und Einkommensklasse. Die Möglichkeiten dieser unterschiedlichen Nachfrage können mit unterschiedlichen Produkteangeboten genutzt werden. Auch dazu können Labels verwendet werden, so lange die Information klar und richtig ist. Das ist einer der Hauptgründe dafür, dass in Zukunft öffentliche und private Labels nebeneinander bestehen werden. Dank der privaten Labels können die komparativen Vorteile insbesondere der grossen Unternehmen genutzt werden, während die weniger strengen öffentlichen Labels es ermöglichen, die meisten Produzenten mitmachen zu lassen (Reardon et al., 2001). Da die Zertifizierungskosten hoch sind, insbesondere für Kleinbetriebe, ist es wichtig, diese durch die Finanzierung gewisser «nicht rentabler» Aktivitäten mit einzubeziehen (Schümperli Younossian, 2001). Die Promotoren von Labelprodukten müssten Strategien nicht nur für deren Produktion 
und Vermarktung sondern auch für die Diversifizierung der Produkte und für die Wertschöpfung durch lokale Verarbeitung und Verpackung ausarbeiten.

Damit zumindest ein Teil der beträchtlichen Wertschöpfung, welche bei der Vermarktung und Verteilung geschaffen wird, den Exporteuren des Südens zugute kommt, müssten diese Spezialitäten und andere Nischenprodukte anbieten und ihr Angebot damit ausweiten. Das könnten Produkte ausserhalb der Saison sein, die zudem den Vorteil haben, das lokale Angebot im Norden nicht zu konkurrenzieren. Wenn Verarbeitung und Verpackung vermehrt im Ursprungsland erledigt werden, kann dies die Marge wesentlich erhöhen. Die dazu erforderlichen Massnahmen können relativ einfach sein wie zum Beispiel das Dörren, oder etwas komplizierter wie die Herstellung von Säften oder frischem Fruchtsalat. Dazu müssen unbedingt spezifische Strategien auf Unternehmensebene eingeführt werden. In Burkina Faso verkaufen die Dörrbetriebe für Mangos zum Beispiel die erste Qualität in Europa und die zweite lokal. Auch die lokalen Ressourcen müssen besser verwertet werden, sowohl auf den lokalen wie auf den regionalen und internationalen Märkten. Es gibt viele Märkte für Früchte, Gewürze, Heilkräuter usw. Dieses so genannte «Nischenmarktvorgehen» ist besonders geeignet für Kleinproduzenten (Ramírez Farías, 2001). Ausserdem kann dadurch die Artenvielfalt erhalten und verwertet werden. Ein Beispiel dafür ist die Initiative TechnoServe, welche die Exporteure und Produzenten zusammen mit einer Forschungsstation geschaffen haben und dank welcher Produktion und Vermarktung einer Erbsenart verbessert werden konnten. Der angestrebte Markt ist ein Nischenmarkt mit hoher Wertschöpfung (frische und verarbeitete Erbsen) in Indien und Europa (TechnoServe, persönliche Mitteilung).

Für jede Initiative ist eine mittel- und langfristige Partnerschaft anzustreben. Barjolle (2001) zeigte auf, dass einige Arten von Zusammenschlüssen zu tieferen Transaktionskosten beitragen können. Partnerschaften, ob handelsbezogen, auf Gegenseitigkeit beruhend, formell oder informell, ermöglichen im Allgemeinen einen besseren Zugang zu Information und Ausbildung. Die Partnerschaft zwischen Produzenten und Importeuren wie beim Max-Havelaar-Label bietet mittelfristig einen sicheren Absatzmarkt. Die Importeure von exotischen Früchten und die Baumwollhändler von Westafrika, meist Spezialisten in ihren Branchen, arbeiten im Allgemeinen auf der Basis einer mittelfristigen Partnerschaft mit verschiedenen Produzentengruppen. Damit können Abkommen geschlossen werden, zum Beispiel über Vorauszahlung, mehrjährige Verträge und Festlegung eines Grundpreises. So haben sich die südafrikanischen Mangoproduzenten mit den Produzenten in Guinea zusammen getan, um die Ernteperiode zu verlängern, ihre Produktion zu diversifizieren und das Know-how in beiden Ländern auszubauen. In Kenia konnte Homegrown dank einer dreiteiligen Partnerschaft in Afrika eine Führerrolle beim Export von frischem, vorverpacktem Gemüse für die Supermärkte in England übernehmen. Zu dieser Partnerschaft gehören die Regierung und internationale Entwicklungsinstitutionen. Es entstehen Zusammenschlüsse von Berufsgruppen wie Produzenten und Verarbeitern, so zum Beispiel in Senegal für Erdnüsse und in Benin für Baumwolle. Einige Industriestaaten unternehmen auch immer mehr, um für ihre Qualitätsprobleme zusammen mit dem Süden eine Lösung zu finden und fangen an, mit diesen zusammenzuspannen. Die Mangoproduzenten in Florida nahmen zum Beispiel Kontakt auf mit jenen in Ägypten, um ihren Pflanzenpool mit Unterstützung einer Entwicklungsorganisation zu verbessern (International Focus, 2000). 
Die Vermarktung und noch mehr der Export erfordern eine immer stärkere Professionalisierung und immer mehr Unternehmergeist. Neuere Studien zeigen auf, dass die Exporteure eher zu Risiken neigen als die exportwilligen Unternehmer (Streeter und Bills, 1998), dass die Grösse der Unternehmen einen Einfluss hat auf ihre Möglichkeiten, zu Informationen zu kommen (Ades und Glaeser, 1999) und sich so auszurüsten, dass sie ein Label erhalten (Reardon et al., 2001). Die Ausbildung zum Unternehmertum muss diese Elemente berücksichtigen. Konkret bedingt das Flexibilität und eine Anpassung der Ausbildungsdauer des Zielpublikums.

Auf der Ebene der Forschung wird auf die unterschiedlichen Mittel zu achten sein, welche herkömmliche und neuere Netze in die Forschung investieren. Diese müsste sich sowohl mit Produktion und Verarbeitung der lokalen Ressourcen für den lokalen wie den internationalen Markt wie mit den nicht herkömmlichen und anderen Nischenprodukten befassen, welche auf den regionalen Märkten im Süden und jenen im Norden Erfolgschancen aufweisen. Es könnten zum Beispiel auch Studien über die Anwendung der Markennamen und der kontrollierten Ursprungsbezeichnungen durchgeführt werden.

Die Informationsströme sollten als Strategieinstrumente für alle Akteure der Nahrungsmittelkette intensiviert werden, um die im wirklichen wie im übertragenen Sinn wachsende Distanz zwischen Produzenten und Konsumenten zu überwinden. Für die Produzenten müssen in den Informationen Preis und Kosten enthalten sein, aber auch die verschiedenen Vermarktungsalternativen, Zahlungsmethoden usw. Ein Beispiel für die bessere Verbreitung der Informationen ist die Initiative der Systèmes d'Information des Marchés en Afrique de l'Ouest, welche ihre Informationen jede Woche in Form eines Bulletins und über ländliche Radiostationen verbreitet.

\section{SCHLUSSBEMERKUNGEN}

Es wird oft gesagt, dass die Bauern und die Verteiler im Süden wie im Norden zwar für soziale und Umweltaspekte empfänglich sind, trotzdem aber lieber kurzfristig ihr Einkommen erhöhen wollen, als die Umwelt langfristig zu schonen. Von Seiten der Konsumenten wird zwar von einer «effizienten Ernährung" gesprochen, welche die einheimische biologische Landwirtschaft im Freiland sowie «faire» Produkte unterstützt (WWF, 2000), gleichzeitig wird den Konsumenten aber vorgeworfen, nur das kurzfristige und billige Vergnügen anzustreben. Liebe Konsumentin, lieber Konsument: was hatten Sie zuletzt auf dem Teller? 


\section{REFERENZEN}

Ades A. und Glaeser E., 1999. Evidence on Growth, Increasing Returns and the Size of the Market. Quarterly Journal of Economics, Vol. 3, Nr. 114, S. 1025-46.

Auroi C. und Schümperli Younoussian C. (Dir.), 2001. Le commerce durable. Vers de plus justes pratiques commerciales entre le Nord et le Sud, Genf, IUED.

Barjolle D., 2001. Hybrid forms of governance: the case of artisanal food products. Beitrag für das 78. EAEE-Seminar, Dänemark, Juli 2001.

CBI und SIPPO (Swiss Import Promotion Programme), 1999. Exporting to Switzerland and the European Union. Rotterdam und Zürich.

Crole-Rees A. und Soulé B.G, 2001. Etude relative au mécanisme de formation des prix de cession de coton graine et des intrants agricoles au Bénin. Rapport final, Vol. I. Lausanne und Cotonou, Oktober 2001 .

Damary P., 2001, «Le label de qualité en agriculture biologique: un pionnier dans le domaine du commerce durable», in Auroi C. und Schümperli Younoussian C. (2001), S. 137-152.

Diopp S. G., 1999. Conditions for food manufactoring in Sub-Saharan Africa. Beitrag für den Workshop über Nahrungsmittel im Afrika südlich der Sahara, in Bamako. CASIN, SG 2000. 15.-19. Oktober 1999.

Evans R.K., 1999. From small farms to supermarkets. In: Breth S.A. (Hrsg.), 1999, Partnerships for rural development in Sub-Saharan Africa. CASIN/SAA/SGGlobal 2000, Genf.

EFTA (European Free Trade Association), 1998. Commerce équitable. Mémento pour l'an 2000. EFTA, Maastricht.

FiBL (Forschungsinstitut für biologischen Landbau) und Sippo, 2001. The organic market and the European Union. Overview and market access information for producers and international trading companies. Januar 2001.

FIDA, 2001. Rapport 2001 sur la pauvreté rurale: comment mettre fin à la pauvreté rurale. FIDA, Rom, September 2001.

Gassmann R., 2001. Chiquita - ein grosser Schritt voraus. Brückenbauer Nr. 14, 3. April 2001.

International Focus, 2000. UF seeks mango exchange with Egypt to improve production. International Focus, Vol. 11, Nr. 2, März-April 2000.

IUED, 2001. Le commerce durable: vers de plus justes pratiques commerciales entre le Nord et le Sud. Genf, Juni 2001.

Lhéraut G., 2001. Logistique d'exportation des pays ACP pour les fruits et légumes et les produits horticoles. Beitrag für das Séminaire africain francophone sur la diversification et le développement du secteur horticole, Bamako, 13.-15. Februar 2001.

Max Havelaar, 1999. Stiftung Max Havelaar. Jahresbericht. Basel, 1999.

Rabobank International, 2001. Fresh Supplies for Asia, Asia Fruit Congress 2001, 9. Mai.

Ramírez Farías L., 2001. Globalisation and livelihood diversification through non-traditional agricultural products: the Mexio case. ODI, Natural Resource Perspective, Nr. 67, Juni 2001.

Reardon Th., Codron J.-M., Busch L. und Harris C., 2001. Global change in agrifood grades and standards: agribusiness strategic responses in developing countries. International Food and Agribusiness Management Review, Vol. 2, Nr. 3-4, S. 421-435.

Roddick A., 1991. Body and Soul. Vermillon, London.

Ronchi L., 2000. Fair trade in Costa Rica: an impact report. University of Sussex, Brighton.

SAMGA (South African Mango Growers' Association), 1999. Safe Quality Mango Management (SQMM): Protokoll. Tzaneen.

Schümperli Younossian C., 2001. «Pourquoi subventionner le commerce équitable». In: Auroi C. und Schümperli Younossian C. (2001), S. 227-249.

Streeter D. und Bills N., 1998. Information strategies for agricultural exporters. International Food and Agribusiness Management Review, Vol. 1, Nr. 1, S. 25-40.

UNCTAD, 2001. Moyens d'accroître la capacité des pays en développement de produire et d'exporter des produits agricoles et alimentaries, y compris des biens très spécialisés, et notamment des produits écologiques. Informationsnote vom Sekretariat der UNCTAD. TD/B/COM. 1 EM. 15/2, Genf, 11. Mai 2001.

Weltbank, 2001. World Development Report 2000/2001. Attacking poverty. Weltbank, Washington DC. WWF, 2000. Der Labelführer. WWF Schweiz, Vernier. 